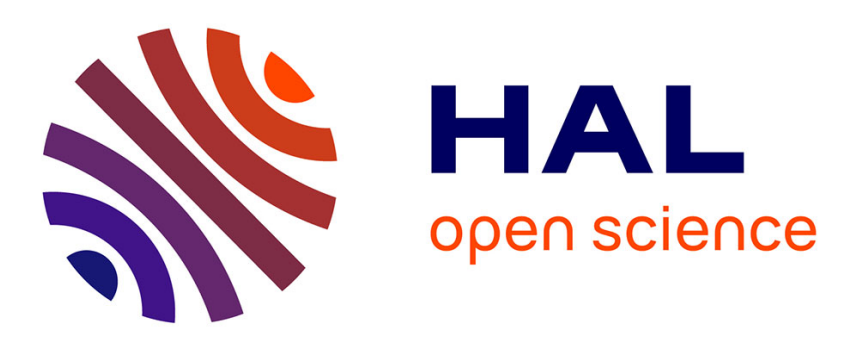

\title{
Dual behavior or coexistence of trapped and free states in reducible rutile $\mathrm{Ti} \mathrm{O} 2$
}

\author{
Jingfeng Li, Stéphane Chenot, Jacques Jupille, Remi Lazzari
}

\section{To cite this version:}

Jingfeng Li, Stéphane Chenot, Jacques Jupille, Remi Lazzari. Dual behavior or coexistence of trapped and free states in reducible rutile Ti O 2. Physical Review B, 2020, 102 (8), 10.1103/PhysRevB.102.081401 . hal-02947128

\section{HAL Id: hal-02947128 \\ https://hal.sorbonne-universite.fr/hal-02947128}

Submitted on 23 Sep 2020

HAL is a multi-disciplinary open access archive for the deposit and dissemination of scientific research documents, whether they are published or not. The documents may come from teaching and research institutions in France or abroad, or from public or private research centers.
L'archive ouverte pluridisciplinaire HAL, est destinée au dépôt et à la diffusion de documents scientifiques de niveau recherche, publiés ou non, émanant des établissements d'enseignement et de recherche français ou étrangers, des laboratoires publics ou privés. 


\title{
Dual behavior or coexistence of trapped and free states in reducible $\mathrm{TiO}_{2}$ rutile
}

\author{
Jingfeng Li, Stéphane Chenot, Jacques Jupille, and Rémi Lazzari* \\ CNRS, Sorbonne Université, Institut des NanoSciences de Paris, \\ UMR 7588, 4 Place Jussieu, F-75005 Paris, France
}

(Dated: September 11, 2020)

\begin{abstract}
Excess electron transport is explored at the reduced $\mathrm{TiO}_{2}(110)$ rutile surface through HighResolution Electron Energy-Loss Spectroscopy. Changes in lineshape, intensity and energy position of elastic and phonon peaks are associated to changes in charge carrier and band gap state excitations. The dielectric modeling points to a defined inward charge profile and to distinct bulk and surface transport properties. The one-to-one correlation between band gap state intensity and carrier density favors a dual polaronic behavior of charge carriers over the coexistence of trapped and free-states.
\end{abstract}

Due to the central importance of the formation of charge carriers and of their bulk/surface transfer in most of the (photo)catalyic properties of titanium dioxide, a lot of effort has gone into understanding the behavior of electrons in this material. Commonly generated by surface oxygen vacancies and titanium interstitials whose formation is favored over a wide thermodynamic range, excess electrons populate $\mathrm{Ti}$ 3d-derived states to form the so-called band gap states (BGS) lying at $0.8-1 \mathrm{eV}$ below the Fermi level ${ }^{1-8}$. They are currently described in the framework of the small-polaron model ${ }^{9-12}$ in which their localization on regular surface and subsurface $\mathrm{Ti}$ sites ${ }^{13-15}$ entails a local distortion of the oxide lattice. The picture is all the more general that similar charge distributions are also evidenced for n-doping with hydrogen ${ }^{16,17}$ or aliovalent cations and anions ${ }^{18}$, electron injection ${ }^{19}$ and UV irradiation ${ }^{17}$, in line with theoretical predictions ${ }^{10,14,20,21}$.

Now, the apparent deep character of the BGS seems to run counter the high bulk mobility observed in the n-type $\mathrm{TiO}_{2}{ }^{22}$, which raises the hotly debated issue of the nature of carriers in titania ${ }^{9-11,22-24}$. The conductivity was first suggested to stem from polaron hopping mechanism with a diffusion barrier of few tens of $\mathrm{meV}^{9,11}$. Due electron-phonon coupling, point defects and doping elements appear as deep donors, when looking at fast vertical transitions that does not allow lattice relaxation, and as shallow donors, when considering thermodynamic transitions involved in transport measurements ${ }^{11}$. Alternatively, arguing that the polaron stability precludes thermal excitation towards delocalized states while a much too high energy is required for polaron hopping, the two states were assumed to coexist thanks to an energy barrier that prevents delocalized electrons from collapsing into the self-trapped polaronic states ${ }^{10,25}$. So far only addressed by theoretical approaches, the issue of charge transport requires an experimental framework. Moreover, focus has been put to date in bulk environment although the transfer to the titania surface of carriers created in bulk is an essential step in the mechanisms at work in most functional applications ${ }^{6}$. In this context, excess electrons in reduced $\mathrm{TiO}_{2}$ are of fundamental significance to applications since they mimic that of photogenerated/injected electrons in dye sensitized solar cells ${ }^{6}$.

The present work aims at an analysis of the electronic structure of defective rutile $\mathrm{TiO}_{2}(110)$ surfaces by High-Resolution Electron Energy-Loss Spectroscopy (HREELS). The method has the capability of investigating at once the existence of Drude-like carriers (transport) and BGS and their interplay ${ }^{26}$ and to probe both the surface and a region deep enough to be representative of the bulk ${ }^{26,27}$. It is shown herein to be a tool of relevance to resolve the controversial issue of the existence of free and trapped states, at the surface and in the bulk of rutile.

The $(1 \times 1)$ contaminant free $\mathrm{TiO}_{2}(110)$ surface was prepared in a classical way by cycles of ion bombardment and annealing at $\sim 1200 \mathrm{~K}$ under ultra-high vacuum. The exposure to oxygen, whose effects allowed to distinguish contributions from surface bridging oxygen vacancies $\left[\mathrm{O}_{b}(\mathrm{vac})\right]$ and titanium interstitials $\left[\mathrm{Ti}_{\text {int }}\right]$ to $\mathrm{BGS}^{27}$, is used to reveal the impact of point defects and the associated excess electrons to the oxide dielectric behavior. The loss spectra collected at $300 \mathrm{~K}$ are shown in Fig. 1. Qualitatively similar data are colected at $100 \mathrm{~K}$ (not shown). Besides the partial healing of $\mathrm{BGS}^{2-8,27}$, the $\mathrm{O}_{2}$ exposure results in a decrease of the quasi-elastic peak $(\mathrm{QEP}) /$ phonon intensity ratio, in particular the one at $\omega_{s p h, 3}=95 \mathrm{meV}$, and in a more apparent high energy asymmetry of the QEP around $10 \mathrm{meV}$ replicated at the foot of the main phonon peak (Figs. 1-a,b and inset). The depth sensitivity of several nanometers of the phonon losses ${ }^{26,27}$ discards the assignment to adsorption of this variation in intensity. The interpretation of the QEP asymmetry as a combination of phonon modes can be ruled out since, unlike observation, it would lead to a disappearance of that feature at $100 \mathrm{~K}$ (not shown).

To account quantitatively for the $\mathrm{O}_{2}$-induced findings in (HR)EELS spectra (Figs. 1-a,b), data were fitted by means of a theoretical analysis ${ }^{26}$ that was previously developed in the framework of the well-established dielectric theory of EELS ${ }^{28-35}$. To model the interplay between carrier and BGS excitations ${ }^{26}$, the substrate dielectric 

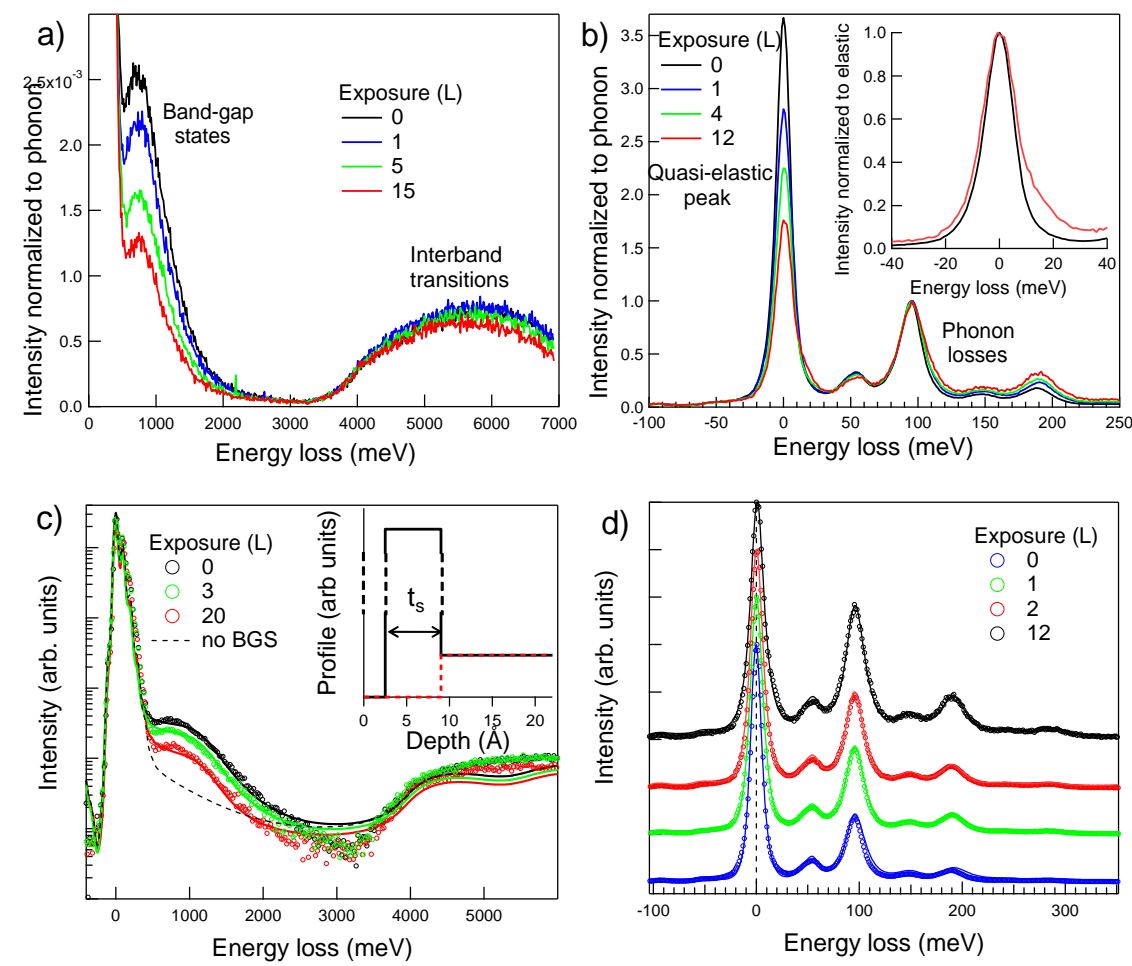

FIG. 1. Effect of $\mathrm{O}_{2}$ exposure (in Langmuir (L): $1 \mathrm{~L}=1.3310^{-6}$ mbar.s) on loss spectra of reduced $\mathrm{TiO}_{2}(110)$ surfaces at $300 \mathrm{~K}$ (incident beam along the $[1 \overline{1} 0]$ direction; incident angle of $\Theta_{I}=60^{\circ}$ ) and modeling: a) EELS (impact energy $E_{I}=38 \mathrm{eV}$; FWHM of quasi-elastic of $50 \mathrm{meV}$ ); b) HREELS $\left(E_{I}=7.5 \mathrm{eV}\right.$; FWHM of $\left.13 \mathrm{meV}\right)$. Intensities were normalized to the main phonon peak $\omega_{s p h, 3}=95 \mathrm{meV}$. The decrease in QEP intensity is more apparent in high than in low resolution. The QEP shape before and at the end of exposure is shown in inset in (b) after normalization to its maximum; c)d) Models (lines) compared to experiments (circles). The inset in (c) shows the used profile of dielectric function of excess electron excitations for reduced (black line) and $\mathrm{O}_{2}$ exposed surface (red line). A thickness $t_{S}=6.5 \AA$ is used in simulations (see text). In Figs. c-d, data were normalized to the QEP and shifted for clarity. For fits, a fixed Gaussian EELS (HREELS, respectively) detector resolution function of $50 \mathrm{meV}$ FWHM $(10 \mathrm{meV})$ and a detector aperture of $\theta_{c}=0.7^{\circ}\left(\theta_{c}=1.2^{\circ}\right)$ were used as determined by specular and out-ofspecular analysis on graphite. function:

$$
\epsilon(\omega)=\epsilon_{P h}(\omega)+\epsilon_{I b}(\omega)+\epsilon_{g s}(\omega)+\epsilon_{P l}(\omega)
$$

involves the tabulated dielectric components of optical phonons $\epsilon_{P h}(\omega)$ and interband transitions $\epsilon_{I b}(\omega)^{36,37}$ and features the excess electrons through their oscillator-like BGS $\epsilon_{g s}(\omega)$ and their Drude-like transport properties $\epsilon_{P l}(\omega)$ given by ${ }^{26}$ :

$$
\epsilon_{g s}(\omega)=\frac{\Omega_{g s}^{2}}{\omega_{g s}^{2}-\omega^{2}-i \Gamma_{g s} \omega} ; \epsilon_{P l}(\omega)=-\frac{\omega_{P}^{2}}{\omega^{2}+i \omega \Gamma_{P}} .
$$

$\left(\Omega_{g s}, \omega_{g s}, \Gamma_{g s},\right)$ stand for the corresponding oscillator strength, frequency and damping, while the plasma frequency $\omega_{P}=\sqrt{n e^{2} / m_{e} m^{*} \epsilon_{0}}$ and damping $\Gamma_{P}=2 \pi e / m_{e} m^{*} \mu$ can be expressed as a function of the carrier density $n$, effective mass $m^{*}$ and mobility $\mu$, $\left(e, m_{e}, \epsilon_{0}\right)$ having their usual meanings. Experimental spectra were fitted with the above parametrization using the generalized EELS dielectric function ${ }^{26,34,35}$ $\xi(\omega)=\epsilon_{\perp}(\omega)$ corresponding to the crystal axis orientation of Figs. 1-a,b with a beam normal to bridging oxygen rows.

Fitting data with flat profiles, i.e. only bulk excitations, failed, especially regarding the QEP/phonon intensity ratio, because leading to a meaningless detector aperture. Therefore, surface and bulk dielectric behaviors were distinguished (in what follows, indexes $S$ and $B$ for all quantities of Eq. 2) via a charge concentration profile $^{27}$ that involves a $2.5 \AA$ thick charge-free surface layer, a layer of thickness $t_{S}$ encompassing excess electrons due to surface defects before reaching the bulk concentration that likely stems from $\mathrm{Ti}_{\text {int }}$ (inset Fig. 1c). The value $t_{S}=6.5 \AA$ (equal to a $\mathrm{TiO}_{2}$ layer spacing along the [110] direction) was determined by a series of fits (not shown). The existence of an electron-rich subsurface region is consistent with observations ${ }^{13,19,27}$ and calculations ${ }^{10,12,14,15,38-40}$. The strategy was to fit first the poorly resolved EELS spectra (Fig. 1-c) that are less sensitive to phonon shifts and intensity variations. Bulk values were obtained at the highest $\mathrm{O}_{2}$ exposure by assuming a complete healing of $\mathrm{O}_{b}(\mathrm{vac})$ surface defects $\left(\Omega_{g s, S}=0\right)$. Then, keeping bulk parameters constant, surface values were obtained at lower $\mathrm{O}_{2}$ exposures. The EELS fit, performed on a logarithmic scale to enhance BGS contribution, was limited to energies lower than $3 \mathrm{eV}$ where long-range dipole interactions with the electron probe dominate with negligible impact scattering ${ }^{26,27}$. Although not included in the fit, interband transitions are nevertheless qualitatively reproduced 
(Fig. 1-c). The bulk $\left(\Omega_{g s, B}, \omega_{g s, B}, \Gamma_{g s, B}\right)$ and surface $\left(\Omega_{g s, S}, \omega_{g s, S}, \Gamma_{g s, S}\right)$ BGS parameters were then fixed to fit the carrier-dependent quantities on HREELS phonon spectra (Fig. 1-d) where the sensitivity to transport properties shows up ${ }^{26}$. High and low $\mathrm{O}_{2}$ exposures were used as above to determine bulk $\left(\omega_{P, B}, \Gamma_{P, B}\right)$ and then surface $\left(\omega_{P, S}, \Gamma_{P, S}\right)$ values, respectively.

The overall good agreement between dielectric models and (HR)EELS scans at $300 \mathrm{~K}$ (Figs. 1-c,d), supports the chosen approach. To ascertain the relevance of the obtained parameters shown in Tab. I, they were one after the other drastically varied in a series of simulations (Fig. 2). On the reduced surface, a large carrier density and therefore plasma frequency $\omega_{P, S}$ and a strong damping $\Gamma_{P, S}$ (as given in Tab. I) are needed to prevent $\omega_{s p h, 3}$ from shifting to higher values $\left(\hbar \omega_{P, S}=0 \mathrm{meV}\right.$ and $\Gamma_{P, S}=10 \mathrm{meV}$; Fig. 2-a); $\omega_{P, S}$ impacts not only the phonon position but also the relative elastic/phonon intensity. On the reoxidized surface (Fig. 2-b), either the absence of bulk carriers $\left(\hbar \omega_{P, B}=0 \mathrm{meV}\right)$ or BGS $\left(\hbar \Omega_{g s, B}=0 \mathrm{meV}\right)$, or the occurrence of strongly damped bulk carriers $\left(\hbar \Gamma_{P, B}=350 \mathrm{meV}\right)$ lead to obvious discrepancies on the $\omega_{s p h, 3}$ position with dramatic amplifications in the multiple and combination modes. Those simulations demonstrate that line shapes are extremely sensitive to dielectric parameters. As previously stressed $^{26}$, phonons are very sensitive reporters of the combined screening of BGS and carrier excitations, in particular that at $95 \mathrm{meV}$ due to its strong oscillator strength. The crucial modelling of the QEP asymmetry and QEP/phonon ratio definitely requires bulk and surface carrier excitations.

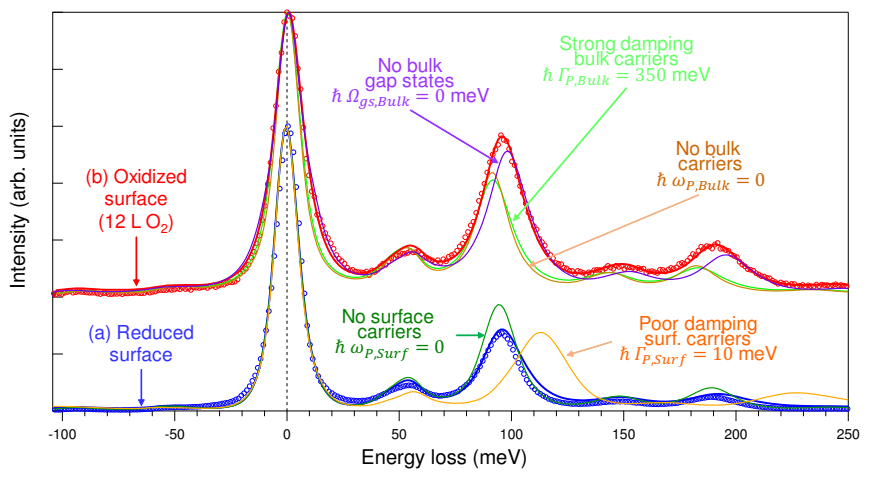

FIG. 2. Comparison between best fits of Fig. 1-d and various simulations: a) reduced surface (0 L; data : blue circles; best fit : blue line) without surface carriers $\left(\hbar \omega_{P, S}=0\right.$, dark green line) or poorly damped surface carriers $\left(\hbar \Gamma_{P, S}=10 \mathrm{meV}\right.$, orange line); b) oxidized surface (12 L; data: red circles; best fit : red line) without the contribution of bulk carriers $\left(\hbar \omega_{P, B}=0\right.$, brown line), or of bulk BGS $\left(\hbar \Omega_{g s, B}=0\right.$, violet line) or with strongly damped bulk plasmon contributions $\left(\hbar \Gamma_{P, B}=350 \mathrm{meV}\right.$, light green line).

The existence of bulk and surface carriers with different transport characteristics and similar BGS signatures is substantiated (Tab. I). While some scattering anisotropy is observed in directions perpendicular or parallel to the c-axis, the observed behaviors are qualitatively similar (not shown). The reliability of the found values (Tab. I) can be further tested by comparison to literature. The decrease by $\sim 5$ of the normalized concentration $n_{B} / m^{*}$ of the bulk carriers between $100 \mathrm{~K}$ and $300 \mathrm{~K}$, derived from the Drude frequency $\omega_{P, B}^{2}=n_{B} e^{2} / m_{e} m^{*} \epsilon_{0}$, matches the evolution of the Hall coefficient $R_{H} \sim 1 / n_{B}{ }^{22,41}$. Taking $m^{*} \simeq 10$ along the perpendicular direction ${ }^{22,41-45}$ leads to a carrier density of $n_{B} \simeq 610^{19} \mathrm{~cm}^{-3}$ at $300 \mathrm{~K}$. Assuming an exhaustion regime at $300 \mathrm{~K}$ for bulk stoichiometry defects, this value sets a lower limit of the degree of reduction $x \simeq 10^{-3}$ in $\mathrm{TiO}_{2-x}$ as expected for the present annealing temperature ${ }^{46}$ and a Fermi level close to the conduction band minimum $\left(E_{C}-E_{F}=64 \pm 5 \mathrm{meV}\right)^{12,47}$. The position of the donor level $E_{C}-E_{D}=30 \pm 10 \mathrm{meV}$ determined via the $n_{B}$ temperature dependence agrees with transport measurements ${ }^{22,48,49}$. Regarding surface carriers, assuming the release of two electrons per $\mathrm{O}_{b}$ (vac), the found plasma frequency $\omega_{P, S}$ (Tab. I) leads to a surface carrier density corresponding to $a \mathrm{O}_{b}$ (vac) coverage of $\sim 10 \%$ of bridging oxygen that agrees with the common consensus ${ }^{1-8}$.

A previous bulk-sensitive infrared reflectivity study of $\epsilon_{\perp}(\omega)^{43}$ (same direction as herein) of rutile at various bulk reduction states revealed a linear link between the bulk plasmon frequency $\omega_{P, B}$ and the high frequency value $\epsilon_{\infty}$ of the phonon dielectric function. Variations of $\epsilon_{\infty}$ were assigned to "transitions in the visible and near-infrared which darken the crystal" ${ }^{43}$. Using BGS position $\omega_{g s, B}$ and damping $\Gamma_{g s, B}$ as found herein, this $\epsilon_{\infty}$-evolution can be traced back to the oscillator strength $\Omega_{g s, B}$ of the present parametrization. Fig. 3-a shows that (HR)EELS and infrared data follow a consistent and similar linear link. Our previous dielectric simulations ${ }^{26}$ indicate that a constant $\omega_{s p h, 3}$ phonon frequency requires a cancellation of upwards (Drude behavior) and downwards (BGS) screening shifts, implying $\Omega_{g s, B}=\sqrt{\alpha_{P} / \alpha_{g s}} \omega_{P, B}$, where $\alpha_{P}, \alpha_{g s}$ are the slopes of phonon shifts versus $\Omega_{g s, B}^{2}$ and $\omega_{P, B}^{2}{ }^{26}$. Using values of Tab. S2 of Ref. 26, one finds $\sqrt{\alpha_{P} / \alpha_{g s}}=11.2$ in agreement with the reinterpreted infrared results $\Omega_{g s, B}=10( \pm 0.2) \omega_{P, B}{ }^{43}$. Now, can excess electrons trapped by a polaronic distortion and more delocalized ones giving rise respectively to BGS and Drude transport behavior coexist? If so, the matching of 100 and $300 \mathrm{~K}$ data on the same line (Fig. 3-a) would point to quite fortuitious similar thermally activated barriers for both type of carriers ${ }^{10}$ to keep $\omega_{P, B} / \Omega_{g s, B}$ constant. The correlation favors instead a dual bulk behavior of bulk excess electrons, that appear as either deep lying states or highly mobile carriers depending on the way and frequency at which they are excited, which is compatible with the polaron theor ${ }^{50,51}$. Interest- 


\begin{tabular}{|c|c|c|c|c|c|c|c|}
\hline & \multicolumn{4}{|c|}{ Plasmon } & \multicolumn{3}{|c|}{ Band gap states } \\
\hline & $\begin{array}{c}\hbar \omega_{P} \\
(\mathrm{meV})\end{array}$ & $\begin{array}{c}n / m^{*} \\
\left(\mathrm{~cm}^{-3} / \mathrm{cm}^{-2}\right)\end{array}$ & $\begin{array}{c}\hbar \Gamma_{P} \\
(\mathrm{meV})\end{array}$ & $\begin{array}{c}\mu \cdot m^{*} \\
\left(\mathrm{~cm}^{2} \cdot \mathrm{V}^{-1} \cdot \mathrm{s}^{-1}\right)\end{array}$ & $\begin{array}{l}\hbar \Omega_{g s} \\
(\mathrm{meV})\end{array}$ & $\begin{array}{c}\hbar \omega_{g s} \\
(\mathrm{meV})\end{array}$ & $\begin{array}{c}\hbar \Gamma_{g s} \\
(\mathrm{meV})\end{array}$ \\
\hline Bulk (300 K) & $92 \pm 10$ & $6.1 \pm 1.310^{18}$ & $\simeq 0 \pm 20$ & $>60$ & $1270 \pm 100$ & $1260 \pm 100$ & $950 \pm 100$ \\
\hline Bulk (100 K) & $40 \pm 10$ & $1.2 \pm 0.610^{18}$ & $\simeq 0 \pm 200$ & $>6$ & $470 \pm 100$ & $1170 \pm 100$ & $760 \pm 100$ \\
\hline Surface $(300 \mathrm{~K})$ & $650 \pm 150$ & $2.0 \pm 1.010^{13}$ & $>500$ & $<2.3$ & $1500 \pm 250$ & $1100 \pm 200$ & $970 \pm 200$ \\
\hline Surface $(100 \mathrm{~K})$ & $330 \pm 150$ & $5.1 \pm 4.610^{12}$ & $>500$ & $<2.3$ & $1150 \pm 250$ & $1150 \pm 200$ & $970 \pm 200$ \\
\hline
\end{tabular}

TABLE I. Fitted bulk and surface dielectric characteristics of excess electrons at 100 and $300 \mathrm{~K}$ (see text). Values are given for a surface layer of $t_{S}=6.5 \AA$. The bulk $n_{B} / m^{*}$ and the surface carrier density $n_{S} / m^{*}$ are deduced from the plasma frequency $\omega_{P}^{2}=n e^{2} / m_{e} m^{*} \epsilon_{0}$. Electron mobilities are obtained from the plasmon damping through $\mu m^{*}=2 \pi e / m_{e} \Gamma$. Surface values are given for the pristine surface (i.e. after vacuum annealing or 0 L-exposure) while bulk values are obtained after the highest $\mathrm{O}_{2}$ exposure. Note that the BGS excitation energy determined in loss experiments differs from the binding energy determined in photoemission $^{6}$.
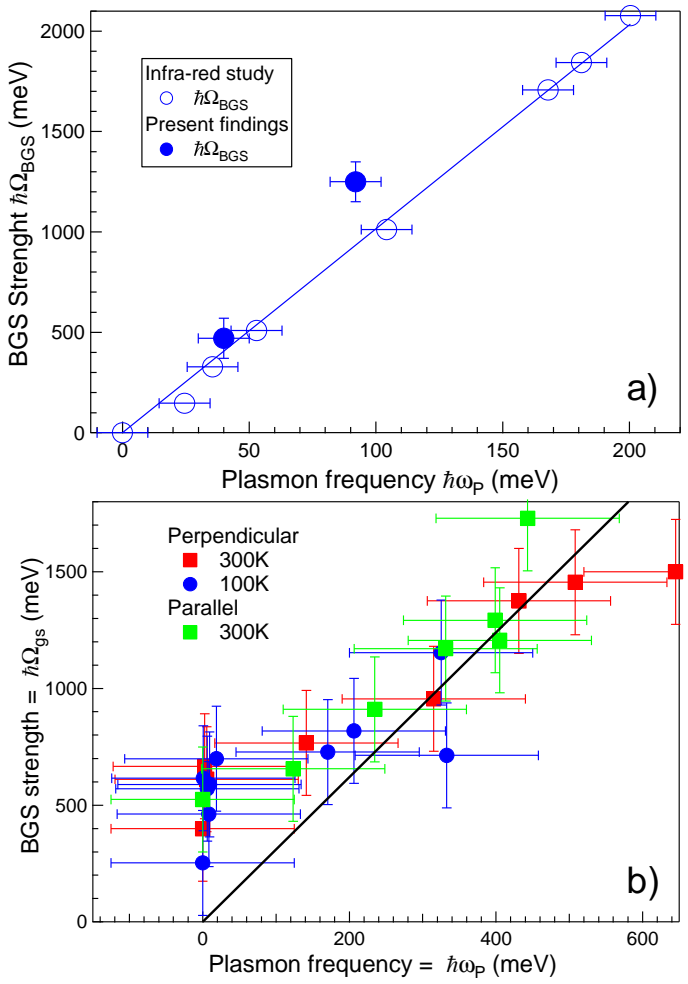

FIG. 3. Correlation between BGS oscillator strength $\Omega_{g s}$ and plasma frequency $\omega_{P}$ : a) Evolution of bulk quantities $\left(\Omega_{g s, B}, \omega_{P, B}\right)$ as rederived from the analysis of infrared data ${ }^{43}$ (open circles). The present (HR)EELS findings for bulk electrons at $100 \mathrm{~K}$ and $300 \mathrm{~K}$ are overlapped with filled symbols; b) Correlation between $\Omega_{g s, S}$ and $\omega_{P, S}$ relative to surface excess electrons for all measurement conditions (temperature and orientation) as indicated in figure. ingly, upon $\mathrm{O}_{2}$ exposure that heals mainly $\mathrm{O}_{b}$ (vac), a similar dual behavior with $\Omega_{g s, S} \simeq 3( \pm 0.3) \omega_{P, S}$ is observed at the rutile surface although changes in electrostatic potential explain changes in slope (Fig. 3-b).

By reflecting a change of electron density, the correlated variations of plasma frequencies and BGS oscillator strengths, both at the surface and in the bulk, strongly favor the polaronic behavior of excess electrons in rutile $^{51}$. Their transport signature, although suspected by molecular dynamics ${ }^{15,39}$, was never evidenced up to now. The predicted apparent hopping activation energy ${ }^{14,15}$ implies a dynamical averaging of the localization of electrons over several adjacent $\mathrm{Ti}$ atoms at $300 \mathrm{~K}$. The present HREELS study shows that $\mathrm{O}_{b}(\mathrm{vac})$ generates a "bidimensional" (or at least over several subsurface $\mathrm{TiO}_{2}$ layers $^{12-15,19,38,39}$ ) confined gas of polarons with a large surface density $\left(n_{S} / m^{*} \simeq 10^{13} \mathrm{~cm}^{-2}\right)$ and an apparent activation energy of $n_{S} / m^{*}$ between 100 and $300 \mathrm{~K}$ close to the bulk value $(\sim 20 \mathrm{meV})$, but with a lower mobility as reflected by damping values (Tab. I). Combined with the previous research on the inward distribution of excess charges ${ }^{27}$, the present study draws an innovative view of the electronic distribution and transport in the surface region of reduced rutile in connexion to the bulk of the oxide.
* Corresponding author :remi.lazzari@insp.jussieu.fr

1 J. Jupille and G. Thornton, Defects at oxide surfaces (Springer, New York, 2015).

2 U. Diebold, Surf. Sci. Rep. 48, 53 (2003).
3 C. Lun Pang, R. Lindsay, and G. Thornton, Chem. Soc. Rev. 37, 2328 (2008).

${ }^{4}$ L.-M. Liu, P. Crawford, and P. Hu, Prog. Surf. Sci. 84, 155 (2009). 
5 Z. Dohnálek, I. Lyubinetsky, and R. Rousseau, Prog. Surf. Sci. 85, 161 (2010).

6 M. A. Henderson, Surf. Sci. Rep. 66, 185 (2011).

7 C. L. Pang, R. Lindsay, and G. Thornton, Chem. Rev. 113, 3887 (2013).

8 M. A. Henderson and I. Lyubinetsky, Chem. Rev. 113, 4428 (2013).

9 N. A. Deskins and M. Dupuis, Phys. Rev. B 75, 195212 (2007).

10 A. Janotti, C. Franchini, J. B. Varley, G. Kresse, and C. G. Van de Walle, Phys. Status Solidi (RRL) 7, 199 (2013).

11 G. Mattioli, P. Alippi, F. Filippone, R. Caminiti, and A. A. Bonapasta, J. Phys. Chem. C 114, 21694 (2010).

12 M. Setvin, C. Franchini, X. Hao, M. Schmid, A. Janotti, M. Kaltak, G. Van de Walle, G. Kresse, and U. Diebold, Phys. Rev. Lett. 113, 086402 (2014).

13 P. Krüger, S. Bourgeois, B. Domenichini, H. Magnan, D. Chandesris, P. Le Fèvre, A. M. Flank, J. Jupille, L. Floreano, A. Cossaro, A. Verdini, and A. Morgante, Phys. Rev. Lett. 100, 055501 (2008).

14 N. A. Deskins, R. Rousseau, and M. Dupuis, J. Phys. Chem. C 113, 14583 (2009).

15 P. M. Kowalski, M. F. Camellone, N. N. Nair, B. Meyer, and D. Marx, Phys. Rev. Lett. 105, 146405 (2010).

16 D. A. Panayotov and J. T. Yates, Chem. Phys. Lett. 436, 204 (2007).

17 H. Sezen, M. Buchholz, A. Nefedov, C. Natzeck, S. Heissler, C. Di Valentin, and C. Wöll, Science Reports 4, 3808 (2014).

18 M. Chiesa, M. Paganini, S. Livraghi, and E. Giamello, Phys. Chem. Chem. Phys. 15, 9435 (2013).

19 P. Krüger, J. Jupille, S. Bourgeois, B. Domenichini, A. Verdini, L. Floreano, and A. Morgante, Phys. Rev. Lett. 108, 126803 (2012).

20 T. Albaret, F. Finocchi, C. Noguera, and A. De Vita, Phys. Rev. B 65, 035402 (2001).

21 P. G. Moses, A. Janotti, C. Franchini, G. Kresse, and C. G. Van de Walle, J. Appl. Phys. 119, 181503 (2016).

22 E. Yagi, R. R. Hasiguti, and M. Aono, Phys. Rev. B 54, 7945 (1996).

23 W.-J. Yin, B. Wen, C. Zhou, A. Selloni, and L. M. Liu, Surf. Sci. Rep. 73, 58 (2018).

24 C. Bigi, Z. Tang, G.-M. Pierantozzi, P. Orgiani, P. K. Das, J. Fujii, I. Vobornik, T. Pincelli, A. Troglia, T. Lee, R. Ciancio, G. Drazic, A. Verdini, A. Regoutz, P. D. C. King, D. Biswas, G. Rossi, G. Panaccione, and A. Selloni, Phys. Rev. Materials 4, 025801 (2020).

25 A. M. Stoneham, J. Gavartin, A. L. Shluger, A. V. Kimmel, D. Muñoz Ramo, H. M. Rønnow, G. Aeppli, and
C. Renner, J. Phys.: Condens. Matter 19, 255208 (2007).

26 R. Lazzari, J. Li, and J. Jupille, Phys. Rev. B 98, 075432 (2018).

27 J. Li, R. Lazzari, S. Chenot, and J. Jupille, Phys. Rev. B 97, 041403(R) (2018).

${ }^{28}$ H. Ibach and D. L. Mills, Electron Energy Loss Spectroscopy and Surface Vibrations, edited by A. P. Inc. (Academic Press Inc., New York, 1982).

29 A. A. Lucas and M. Sunjić, Phys. Rev. Lett. 26, 229 (1971).

30 A. A. Lucas and M. Sunjić, Prog. Surf. Sci. 2, 75 (1972).

31 E. Evans and D. L. Mills, Phys. Rev. B 5, 4126 (1972).

32 E. Evans and D. L. Mills, Phys. Rev. B 7, 853 (1973).

33 D. L. Mills, Surf. Sci. 48, 59 (1975).

34 A. A. Lucas and J. P. Vigneron, Solid. Stat. Commun. 49, 327 (1984).

35 P. Lambin, J. P. Vigneron, and A. A. Lucas, Phys. Rev. B 32, 8203 (1985).

36 D. Schöche, T. Hofmann, R. Korlacki, T. Tiwald, and M. Schubert, J. Appl. Phys. 113, 164102 (2013).

37 E. D. Palik, Handbook of Optical Constants of Solids, Vol. 1-3 (Academic Press, 1985).

38 T. Minato, Y. Sainoo, Y. Kim, H. S. Kato, K. Aika, M. Kawai, J. Zhao, H. Petek, T. Huang, W. He, W. Bing, W. Zhuo, Y. Zhao, J. Yang, and J. G. Hou, J. Chem. Phys. 130, 124502 (2009).

39 C. M. Yim, M. B. Watkins, M. J. Wolf, C. L. Pang, K. Hermansson, and G. Thornton, Phys. Rev. Lett. 117, 116402 (2016).

40 K. Morita, T. Shibuya, and K. Yasuoka, J. Phys. Chem. C 121, 1602 (2017).

41 V. Cristea and V. Babes, Physica Status Solidi A 45, 617 (1978)

42 G. A. Acket and J. Volger, Physica 32, 1680 (1966).

43 J. F. Baumard and F. Gervais, Phys. Rev. B 15, 2316 (1977).

44 E. Hendry, F. Wang, J. Shan, T. F. Heinz, and M. Bonn, Phys. Rev. B 69, 081101(R) (2004).

45 E. Hendry, M. Koeberg, J. Pijpers, and M. Bonn, Phys. Rev. B 75, 233202 (2007).

46 M. A. Henderson, W. S. Epling, C. L. Perkins, C. H. F. Peden, and U. Diebold, J. Phys. Chem. B 103, 5328 (1999).

47 A. K. See, M. Thayer, and R. A. Bartynski, Phys. Rev. B 47, 13722 (1993).

48 L. Hollander and P. L. Castro, Phys. Rev. 119, 1882 (1960).

49 E. Iguchi, K. Yajima, T. Asahina, and Y. Kanamori, J. Phys. Chem. Solids 35, 597 (1974).

50 A. S. Alexandrox and J. Devreese, Advances in polaron physics (Spinger, New York, 2010).

51 D. Emin, Phys. Rev. B 48, 13691 (1993). 